\title{
O esquema corporal de crianças com dificuldade de aprendizagem
}

\author{
Francisco Rosa Neto \\ Kassandra Nunes Amaro \\ Daniela Bosquerolli Prestes \\ Claudia Arab
}

\begin{abstract}
Resumo
Reconhecendo a importância do esquema corporal para o desenvolvimento global da criança, o objetivo desta pesquisa foi verificar o desenvolvimento do esquema corporal de escolares na faixa etária de 6 a 10 anos com queixa de dificuldades de aprendizagem, encaminhados ao Núcleo de Avaliação e Intervenção Motora - NAIM/LADEHU/UDESC. Participaram do estudo 39 crianças, sendo 24 meninos e 15 meninas, provenientes de escolas públicas de Florianópolis. O esquema corporal foi avaliado através da Escala de Desenvolvimento Motor - EDM. Todos os participantes apresentaram atraso no desenvolvimento da Idade Motora Geral e na Idade Motora do Esquema Corporal. O déficit aumentou conforme a idade cronológica dos participantes e a classificação do Quociente Motor Geral e do Quociente Motor do Esquema Coporal do grupo foi Muito Inferior. Os dados sugerem que o desenvolvimento das capacidades motoras, em especial do esquema corporal, são fundamentais para o desenvolvimento de habilidades essenciais à aprendizagem escolar.
\end{abstract}

Palavras- chave: Desenvolvimento motor, esquema corporal, distúrbios da aprendizagem.

\section{The body scheme of children with learning disability}

\begin{abstract}
Recognizing the importance of body scheme for the overall development of the child, our goal, in this work was to investigate the development of body scheme for students aged 6 to 10 years with complaints of learning difficulties, referred to the Division of Motor Assessment and Intervention - NAIM / LADEHU / UDESC. The study included 39 children, 24 boys and 15 girls from public schools of Florianopolis, Southern Brazil. The body scheme was assessed by the Scale of Motor Development - EDM. All participants showed delayed development of General Motor Age and Motor Age of Body Scheme. The deficit increased with the chronological age of the participants and the classification of General Motor Quotient and Motor Quotient of Body Scheme was much lower. The data suggest that the development of motor skills, body scheme in particular, are fundamental to the development of essential skills to academic learning.
\end{abstract}

Keywords: Motor development, body schema, learning disabilities.

\section{El esquema corporal de niños con dificultad de aprendizaje}

\section{Resumen}

Reconociendo la importancia del esquema corporal para el desarrollo global del niño, el objetivo de esta investigación fue verificar el desarrollo del esquema corporal de escolares de 6 a 10 años con queja de dificultades de aprendizaje, encaminados al Núcleo de Evaluación e Intervención Motora - NAIM/LADEHU/UDESC. Participaron del estudio 39 niños, siendo 24 niños y 15 niñas, provenientes de escuelas públicas de Florianópolis. El esquema corporal fue evaluado a través de la Escala de Desarrollo Motor - EDM. Todos los participantes presentaron retraso en el desarrollo de la Edad Motora General y en la Edad Motora del Esquema Corporal. El déficit aumentó conforme la edad cronológica de los participantes, y la clasificación del Cociente Motor General y del Cociente Motor del Esquema Corporal del grupo fue Muy Inferior. Los datos sugieren que el desarrollo de las capacidades motoras, en especial del esquema corporal, son fundamentales para o desarrollo de habilidades esenciales al aprendizaje escolar.

Palabras Clave: Desarrollo motor, esquema corporal, trastornos del aprendizaje. 


\section{Introdução}

Problemas na aquisição da leitura e escrita atingem de forma severa entre $5 \%$ e $10 \%$ das crianças nos primeiros anos do ensino fundamental e chegam a $25 \%$ se considerados os distúrbios leves (Capovilla, Capovilla, Trevisan, \& Rezende, 2006). A dificuldade na aprendizagem pode ser considerada como a interação de uma série de fatores que resultam no baixo rendimento frente à situação de aprendizagem (Medeiros, Loureiro, Linhares, Maturano, 2000). Há muita controvérsia em torno do conceito "dificuldade de aprendizagem", contudo existe um consenso sobre a existência de uma discrepância severa entre o que é esperado academicamente e o desempenho apresentado (Pacheco, 2005).

Muitas pesquisas foram realizadas sobre as perturbações do desenvolvimento, e seus resultados apontam para a relação entre o desenvolvimento das capacidades motoras e o desempenho no processo de aprendizagem escolar da leitura e escrita (Amaro, 2010; Amaro, Brusamarello, Campos, Corazza, \& Xavier, 2008; Amaro, Xavier, Corazza, Brusamarello, \& Rosa Neto, 2009; Beresford, Queiroz, \& Nogueira, 2002; Ferreira, 2007; Freitas, 2008; Medina, Rosa, \& Marques, 2006; Medina-Papst \& Marques, 2010; Neto e cols., 2007; Rosa Neto, Poeta, Silva, \& Silva, 2006). Ferreira, Nascimento, Apolinário e Freudenheim (2006) afirmam que cerca de 5 a $10 \%$ das crianças nos primeiros quatro anos escolares apresentam problemas no desenvolvimento motor.

O desenvolvimento motor é caracterizado por uma série de mudanças que ocorrem ao longo da vida do indivíduo, resultante da interação e necessidades de sua biologia, da tarefa e das condições do ambiente. A participação ativa em atividades motoras é um modo efetivo de reforçar as habilidades essenciais ao raciocínio e a aprendizagem dos conceitos acadêmicos (Gallahue \& Ozmun, 2005). Nas fases iniciais do processo de desenvolvimento motor, alguns elementos são essenciais para a aquisição de padrões fundamentais de movimento, como consciência corporal, direcional e espacial, sincronia, ritmo e sequência de movimento (Gallahue, 2000). Estes aspectos estão plenamente interligados e, quando trabalhados de forma adequada, contribuirão para o desenvolvimento integral da criança, possibilitando-Ihe atuar de forma eficiente no aprendizado de tarefas pertencentes a diversas áreas (Vieira, Santos, Vieira, \& Oliveira, 2004).

De acordo com Tavares (2003), imagem corporal engloba todas as formas pelas quais uma pessoa experimenta e conceitua seu próprio corpo, estando ligada a uma organização cerebral integrada, influenciada por fatores sensoriais, processo de desenvolvimento e aspectos psicodinâmicos. A imagem corporal deve ser compreendida como um fenômeno singular, estruturado no contexto da experiência existencial e individual do ser humano, em um universo de inter-relações entre imagens corporais. Vayer e Destrooper (1979) descrevem imagem corporal como resultado complexo de toda atividade cinética, sendo a imagem do corpo a síntese de todas as mensagens, de todos os estímulos e de todas as ações que permitam à criança diferenciar-se do mundo exterior, e de fazer do "eu" o sujeito de sua própria existência. A imagem do corpo representa uma forma de equilíbrio que, como núcleo central da personalidade, organiza-se em um contexto de relações mútuas do organismo e do meio. Maturana (2004) explica que, em estudos da Neurologia e Psicologia, o termo "imagem corporal" é comumente permutado pelo termo "esquema do corpo" e que existem resistências a algumas definições e confusões na metodologia e conceitualização. Amaro (2010) afirma que, para a Psicologia moderna, a noção do corpo é definida como esquema corporal e envolve o reconhecimento do corpo, de postura correta e do posicionamento dos seus segmentos. O esquema corporal é resultado da união de várias informações obtidas pelas inúmeras experiências corporais, relaciona-se à atividade motora e revela-se pelo movimento e no movimento. O termo "esquema corporal" foi adotado em razão desta ser a nomenclatura utilizada na avaliação motora empregada nesta pesquisa e presente na maior parte dos estudos consultados.

Freitas (2008) refere que a representação que a criança possui do seu próprio corpo é um elemento indispensável na formação de sua personalidade. Para a autora, estudar o corpo, o esquema corporal e imagem corporal é particularmente importante àqueles que se dedicam ao estudo da cognição humana, do ensino e da aprendizagem. Medina, Rosa e Marques (2006) sugerem que os problemas de aprendizagem, na maioria das crianças, não são associados a algum tipo de deficiência mental. A avaliação das capacidades desenvolvidas é essencial para diagnosticar problemas motores e suas consequências, fornecendo dados que viabilizem sugestões de intervenções que favoreçam o desenvolvimento global da criança em todos os seus aspectos (Medina e cols., 2006; Medina-Papst \& Marques, 2010).

Reconhecendo a importância das capacidades motoras para o desenvolvimento global da criança, em especial o esquema corporal, o objetivo desta pesquisa foi investigar o desenvolvimento motor na área do esquema corporal de escolares na faixa etária de 6 a 10 anos que apresentam dificuldades de aprendizagem sem diagnóstico de deficiência física ou mental associada. Todas as crianças foram encaminhadas ao Núcleo de Avaliação e Intervenção Motora - NAIM do Laboratório de Desenvolvimento Humano - LADEHU, da Universidade Estadual de Santa Catarina - UDESC, por queixa de dificuldades de aprendizagem.

\section{Método}

Este estudo consiste em uma pesquisa descritiva, diagnóstica, cuja amostra compreende 39 crianças (24 meninos e 15 meninas) na faixa etária de 6 a 10 anos, provenientes de escolas públicas de diferentes bairros do município de Florianópolis/SC. Os escolares foram encaminhados por profissionais da Saúde ou Educação para ava- 
Tab ela 1. Dados descritivos do desenvolvimento motor geral e do esquema corporal do grupo de crianças com dificuldade de aprendizagem.

\begin{tabular}{cccccc}
\hline & N & $\begin{array}{c}\text { Valor } \\
\text { Minímo }\end{array}$ & Valor & Média & Desvio \\
& & 71 & 131 & $(104,49$ & 18,379 \\
\hline IC & 39 & 24 & 108 & 69,23 & 17,294 \\
\hline IM4 & 39 & 21 & 98 & 71,95 & 17,142 \\
\hline IMG & 39 & -103 & -9 & $-32,54$ & 19,487 \\
IN & 39 & 24 & 93 & 67,38 & 16,314 \\
\hline QM4 & 39 & 20 & 88 & 69,90 & 15,042 \\
\hline QMG & 39 & & & \\
\hline
\end{tabular}

Legenda: idade cronológica (IC); idade motora geral (IMG); idade motora do esquema corporal (IM4); idades negativa (IN), quociente motor do esquema corporal (QM4) e; quociente motor geral (QMG).

liação no Núcleo de Avaliação e Intervenção Motora - NAIM do Laboratório de Desenvolvimento Humano - LADEHU da Universidade do Estado de Santa Catarina - UDESC.

De acordo com os princípios da Ética em Pesquisa, todos os participantes e seus responsáveis foram informados sobre os procedimentos e sobre a possibilidade de deixar a pesquisa a qualquer momento, sem nenhum tipo de prejuízo. Após a autorização dos responsáveis e acompanhadas por eles, as crianças realizaram a avaliação individualmente em sala ampla, silenciosa, arejada e iluminada.

$\mathrm{O}$ instrumento utilizado para avaliação foi a Escala de Desenvolvimento Motor - "EDM" criada por Rosa Neto (2002), que abrange baterias de testes para diferentes áreas do desenvolvimento motor: motricidade fina; motricidade global; equilíbrio; esquema corporal; organização espacial e temporal; e lateralidade. Para fins deste estudo, optou-se por investigar o desempenho dos escolares na área de esquema corporal (IM4). Esta bateria consiste em três testes motores, distribuídos entre 2 e 11 anos, organizados progressivamente em grau de complexidade, sendo atribuído para cada tarefa, em caso de êxito, um valor correspondente à idade motora (IM), expressa em meses. O teste é interrompido quando a criança não concluir a tarefa com êxito, conforme protocolo. Ao final da aplicação, é atribuída à criança uma determinada idade motora. A soma da bateria do esquema corporal com as demais baterias resulta na idade motora geral (IMG) e permite calcular o quociente motor geral (QMG) que classifica os resultados em Muito Superior, Superior, Normal Alto, Normal Médio, Normal Baixo, Inferior e Muito Inferior para o desenvolvimento motor geral.

Os testes de esquema corporal envolvem tarefas de controle sobre o próprio corpo e rapidez: imitação de gestos simples com as mãos e imitação de gestos simples com os braços. Nos dois primeiros testes, a criança fica em pé diante do examinador, que fica sentado para colocar suas mãos em posição neutra entre cada gesto. A criança deve imitar cada gesto das mãos e braços que o examinador realiza. O número de acertos dos gestos corresponde à idade motora entre dois e cinco anos. O terceiro teste, de rapidez, compreende uma folha de papel quadriculado com $25 \mathrm{~cm} x$ $18 \mathrm{~cm}$ (quadrado de $1 \mathrm{~cm}$ de lado), lápis preto e cronômetro. A criança deve fazer um risco em cada quadrado o mais rápido possível em um minuto. A quantidade de traços feitos corresponde à idade motora entre seis e 11 anos.

Para melhor investigar e compreender o desenvolvimento do esquema corporal, os sujeitos da pesquisa foram divididos por faixa etária, havendo 6 crianças no grupo de 6 anos (G6), 8 no grupo de 7 anos (G7), 7 no grupo de 8 anos (G8), 8 no grupo de 9 anos (G9) e 10 no grupo de 10 anos (G10).

Os dados foram armazenados no programa SPSS for Windows $13.0 \mathrm{e}$, para análise estatística, foi feita a distribuição de frequência simples e percentuais, média, variância, valor mínimo e valor máximo, comparando-se os resultados obtidos entre os grupos. Para comparar médias, foi utilizado o teste " $\mathrm{t}$ " para uma amostra, $\operatorname{com} p=0,05$.

\section{Resultados}

Conforme o apresentado na Tabela 1, verifica-se que tanto a Idade Motora Geral (IMG) como a Idade Motora do Esquema Corporal (IM4) estão muito aquém da Idade Cronológica, e que tanto o Quociente Motor Geral (QMG) quanto o Quociente Motor do Esquema Coporal (QM4) apresentam valores que, de acordo com a EDM, são classificados como Muito Inferiores.

O Quociente Motor 4, relativo ao esquema corporal, é obtido pela divisão entre a Idade Motora 4 e a Idade Cronológica, cujo resultado é multiplicado por 100. O Quociente Motor Geral é resultante da divisão entre a Idade Motora Geral pela Idade Cronológica e multiplicado por 100. Em ambos, a classificação do Quociente Motor é dividida em sete níveis: 69 ou menos: Muito Inferior; 70-79: Inferior; 8089: Normal Baixo; 90-109: Normal Médio; 110-119: Normal Alto; 120-129: Superior; 130 ou mais: Muito Superior. 


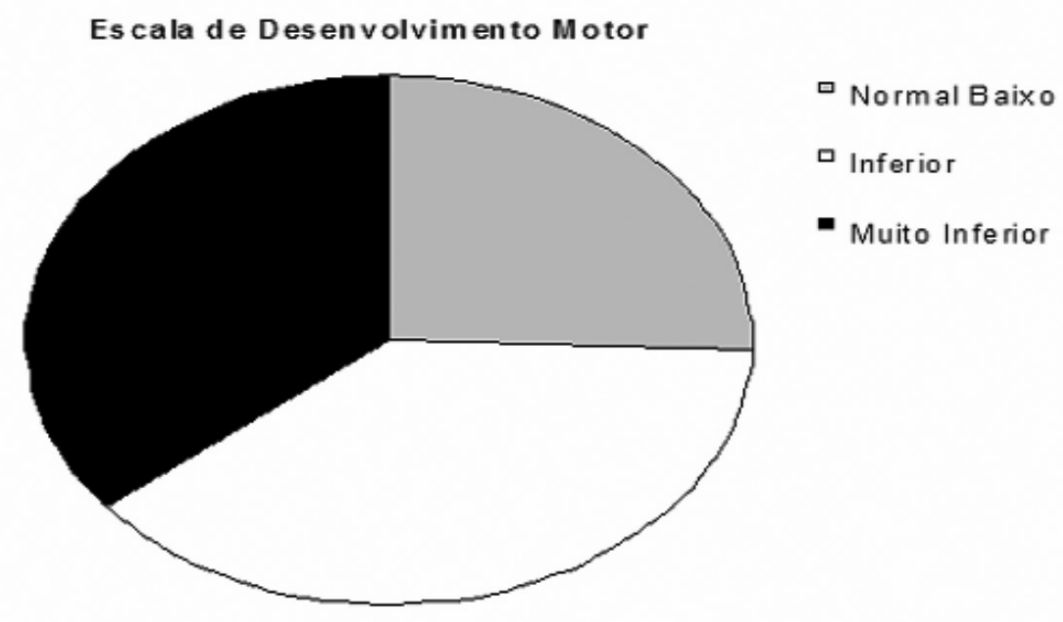

Figura 1. Distribuição da classificação da Escala de D esenvolvim ento M otor.

Tabela 2. Médias da idade cronológica (IC), idade motora geral (IMG), idade motora do esquema corporal (IMA), idades negativa (IN), quociente motor do esquema corporal (QM4) e quociente motor geral (QMG), por grupo etário.

\begin{tabular}{lccccc}
\hline & 6 anos & 7 anos & 8 anos & 9 anos & 10 anos \\
\hline IC & 77,67 & 91,13 & 102,86 & 114,38 & 125,40 \\
\hline IM4 & 64,00 & 61,50 & 66,86 & 69,00 & 79,20 \\
IMG & 61,33 & 65,88 & 68,71 & 79,13 & 79,20 \\
\hline IN & $-16,33$ & $-25,25$ & $-34,14$ & $-35,25$ & $-46,20$ \\
\hline QM4 & 82,33 & 67,13 & 65,00 & 60,25 & 63,30 \\
\hline QMG & 82,33 & 68,50 & 54,29 & 60,38 & 63,40 \\
\hline
\end{tabular}

A Figura 1 evidencia que a grande maioria da amostra teve o desenvolvimento motor classificado como Muito Inferior e Inferior, e nenhuma criança atingiu níveis além do Normal Baixo.

De acordo com a Tabela 2, verifica-se que tanto a IMG como a IM4 parecem manter-se, enquanto a IC aumenta, o que faz com que a Idade Negativa (IN) aumente de grupo para grupo. Nota-se, ainda, que, apenas para o grupo G6, tanto o QMG quanto o QM4 apresentaram valores acima de 80 , ou seja, classificados como Normal Baixo, enquanto que, para os outros cinco grupos, os valores podem ser classificados como Muito Inferior.

Outro fato relevante é que, para o G6, encontrou-se que $33,3 \%$ da amostra têm desenvolvimento do esquema corporal Inferior. No G7, 62,5\% das crianças apresentaram esquema corporal classificado como Muito Inferior e $25 \%$, como Inferior. Já para G8, 42,9\% foi Inferior e 28,6\%, Muito Inferior. Expressivamente, encontraram-se $100 \%$ dos escola- res do G9 com muito atraso no esquema corporal, sendo que $87,5 \%$ dos casos foram Muito Inferior e 12,5\%, Inferior. Finalmente, para o G10, 80\% dos escolares classificaram-se em nível Muito Inferior e 10\%, Inferior. Para todos os grupos, não foram encontradas classificações acima de Normal Baixo.

Segundo o teste T para uma amostra, a diferença foi significativa $(0,000)$ entre a IC e IM4 em todos os grupos.

\section{Discussão}

É essencial considerar diversos fatores quando se trata de estudar as dificuldades de aprendizagem, porque podem envolver aspectos neurológicos, ambientais e sociais, entre outros. Todas as interações, papéis sociais e imagem, constituídos na família, comunidade, escola e demais contextos sociais interferem na aquisição de crenças, valores e conteúdos escolares (Amaro, 2010; Pacheco, 2005). Além 
disso, sabe-se que componentes da aprendizagem motora exercem influências significativas na aquisição de habilidades de aprendizagem cognitiva, pois estas capacidades serão solicitadas posteriormente no processo de aprendizagem da leitura e escrita (Freitas, 2008; Medina e cols., 2006; Medina-Papst \& Marques, 2010). A imagem do corpo, conceituada como esquema corporal, é uma forma equilibrada do núcleo central da personalidade, que se desenvolve entre as relações do sujeito e o meio. A constituição do esquema corporal é fundamental para o desenvolvimento da criança, já que esta organização é uma referência para as diversas possibilidades de ação (Rosa Neto, 2002).

Vários autores encontraram, em seus estudos, déficit motor em escolares de diferentes faixas etárias. Na sua maioria, o esquema corporal esteve entre a principal ou as principais áreas que apresentaram maior déficit motor em relação ao esperado para a idade da criança. Batistella (2001) avaliou 200 escolares de Cruz Alta/RS, de ambos os gêneros, com idades entre seis e 10 anos, que frequentavam as séries iniciais do ensino fundamental de uma escola estadual. Sua amostra alcançou classificação Normal Médio para o Quociente Motor Geral (QMG) e Quociente Motor do Esquema Corporal (QM4). O mesmo resultado foi encontrado por Silva, Rosa Neto, Almeida, Amaro e Silva (2006) em seu estudo com 88 escolares entre seis e oito anos da rede pública municipal de Florianópolis/SC. Pazin (2001) investigou 308 crianças, com idades de oito e nove anos, de escolas em São José/SC, dividindo-as em dois grupos, um para os que apresentaram aptidão física superior e o outro abrangendo os escolares com aptidão física inferior. Enquanto o primeiro grupo obteve classificação Normal Médio para QMG e QM4, o segundo grupo apresentou Normal Baixo. Neste estudo, Pazin refere que as crianças com aptidão física inferior apresentavam também maiores dificuldades na aprendizagem e de motricidade.

Melo (1997) verificou o perfil motor de alunos atletas de oito a 10 anos que praticam regularmente natação, encontrando o resultado de Normal Baixo para o QMG e QM4. Franceschi (1997) avaliou 30 crianças entre oito e 10 anos de idade praticantes de futebol de salão nos clubes e colégio de Florianópolis cujo QM4 foi classificado como Inferior enquanto o QMG foi Normal Baixo.

Costa (2001) avaliou 105 crianças na faixa etária entre cinco e 14 anos, encaminhadas ao Núcleo Desenvolver do Hospital Universitário por queixa de dificuldade de aprendizagem. A classificação do QMG da amostra foi Inferior e a do QM4 foi de Muito Inferior. Fiates (2001) apresentou os mesmos resultados para QMG e QM4 investigando 24 escolares com idades de quatro a sete anos da rede privada de ensino. Houve diferença entre os gêneros, pois o resultado do QM4 foi ligeiramente superior para as meninas (Inferior), enquanto que, para os meninos, foi Muito Inferior. No referido estudo, enfatiza que tal diferença possa ser pelas aulas de balé e dança praticada pela maioria daquela amostra.

Rosa Neto, Poeta, Coquerel e Silva (2004) avaliaram 51 crianças entre quatro e 12 anos com dificuldade de aprendizagem encaminhadas ao programa de psicomotricidade da UDESC. O grupo foi classificado como Inferior para o QMG e QM4. Mello, Poeta e Rosa Neto (2003) encontraram a mesma classificação no estudo com 52 alunos que tinham diagnóstico de Transtorno de Déficit de Atenção e Hiperatividade (TDAH) e eram de ambos os gêneros, com idade entre seis e 10 anos, e matriculados nos anos iniciais do ensino fundamental.

Comparando os dados explanados pelos diferentes autores, é possível perceber que as crianças com dificuldade de aprendizagem e indicativos de TDAH são as que apresentam menores escores na avaliação motora. Os autores que investigaram a relação entre o desenvolvimento motor e a prática de exercícios ou esportes de crianças apresentaram resultados ligeiramente superiores, conforme pode ser visto na Tabela 3. Tais observações sugerem que a prática de atividade física influencia positivamente o desenvolvimento do esquema corporal, assim como a percepção e uso mais apurado deste pode favorecer o desempenho nas atividades escolares.

Baseados nos resultados encontrados, Caetano, Silveira e Gobbi (2005) concluíram que as capacidades envolvendo o esquema corporal das crianças investigadas

Tab ela 3. Tabela comparativa sobre estudos de diferentes autores.

\section{AUTORES}

Estudo atual

Medina-Papst e cols. (2010)

Silva e cols. (2006)

Rosa Neto e cols. (2004)

Mello e cols. (2003)

Costa (2001)

Fiates (2001)

Batistella (2001)

Pazin (2001)

Franceschi (1997)

Melo (1997)
Poe th (2005)

\section{AMOSTRA}

Com DA

Com DA

Escolares

Com TDAH

Com DA

TDAH

Com DA

Escolares

Escolares

Escolares

Praticantes de Futebol

Praticantes de Natação

\begin{tabular}{cl} 
CLASSIFICAÇÃ O & CLA SSIFICAÇÃ O \\
QM G & \multicolumn{1}{c}{ QM 4 } \\
Muito Inferior & Muito Inferior \\
Normal Baixo & Normal Baixo \\
Normal Baixo & Normal Baixo \\
Inferior & Normal Baixo \\
Inferior & Inferior \\
Inferior & Inferior \\
Inferior & Muito Inferior \\
Inferior & Muito Inferior \\
Normal Médio & Normal Médio \\
Normal Médio & Normal Médio \\
Normal Baixo & Normal Baixo \\
Normal Baixo & Inferior \\
Normal Baixo & Normal Baixo
\end{tabular}

Legenda: D A - dificuldade de aprendizagem; TD A H - Transtorno de déficit de atenção e hiperatividade. 
desenvolveram-se por volta dos quatro ou cinco anos de idade e permaneceram no mesmo nível até os seis ou sete anos. Os dados da atual pesquisa demonstram alguma relação com o achado por Caetano e cols. (2005), Medina e cols. (2006) e Medina- Papst e Marques (2010) quando todos os grupos apresentaram déficit motor, agravando-se conforme o aumento da idade cronológica.

Estes dados permitem afirmar que as crianças mais velhas possuem maior dificuldade em desempenhar tarefas que envolvam o controle e reconhecimento do próprio corpo em relação ao esperado para sua idade. Talvez este aumento conforme a idade deva-se à diminuição de situações que lhe permitam explorar seu próprio corpo e o espaço em sua volta. Assim que entram na escola, e conforme os anos acadêmicos se sucedem, as crianças são cada vez mais envolvidas em atividades de sala de aula, sendo menos disponibilizadas a brincadeiras, jogos e atividades que thes facilitem o desenvolvimento da motricidade. Crippa e Souza (2002) relacionaram o atraso no desenvolvimento do esquema corporal em relação à idade cronológica encontrado na sua pesquisa ao excessivo tempo despendido com jogos televisivos e à restrição de atividades de respiração, simetria corporal, equilíbrio postural etc.

Em relação à diferença entre o QMG e o QM4, apenas Poeta (2005) encontrou, em sua amostra de 31 crianças com indicadores de TDAH, resultado para QM4 (Normal Baixo) superior ao QMG (Inferior). Nas demais pesquisas, o Quociente Motor do Esquema Corporal (QM4) apresentou resultados mais baixos do que o QMG, quando não igual. $O$ presente estudo compartilha os mesmos resultados para as idades de sete, oito, nove e 10 anos, quando verificou o QM4 inferior à classificação do QMG. Pressupõe-se que o atraso motor no reconhecimento das partes do corpo e seu uso no espaço e tempo podem ter contribuído para o baixo resultado encontrado nas avaliações motoras. Estas conclusões corroboram com diferentes autores (Beresford e cols., 2002; Cratty, 1964; Fonseca, 1983; Harrow, 1983; Medina e cols., 2006; Scagliotta, 1970) quando atribuem ao desenvolvimento do esquema corporal papel fundamental no desempenho de aprendizagens posteriores.

Todos os escolares participantes do núcleo de avaliação que originou esta pesquisa foram encaminhados com queixa de dificuldade de aprendizagem e todos eles apresentaram atraso motor em relação à idade cronológica.

Os resultados encontrados nestes estudos sugerem relação próxima entre o desenvolvimento do esquema corporal e dificuldades de aprendizagem. A percepção do próprio corpo e a percepção deste no espaço e no tempo são essenciais para o desenvolvimento harmonioso dos aspectos motores, físicos e cognitivos. As dificuldades motoras podem interferir nas relações sociais, emocionais e escolares, por outro lado, explorar o movimento e o brincar espontaneamente precedem as atividades mais estruturadas de aprendizagem. $\mathrm{O}$ estudo mostrou que o déficit relacionado ao esquema corporal aumentou conforme a idade cronológica. Este dado é relevante e remete-nos a refletir sobre a avaliação precoce do desenvolvimento motor no sentido de diagnosticar o problema e planejar intervenção adequada que facilite o processo de escolarização e desenvolvimento do indivíduo como um todo, para que este não chegue aos anos seguintes com déficits motores e de aprendizagem cada vez maiores.

O presente estudo abordou, mais especificamente, o desenvolvimento do esquema corporal, contudo isso não significa que os demais componentes do desenvolvimento motor exerçam papel menos relevante ao desenvolvimento global do sujeito. Tampouco se pretendeu supor que os componentes motores sobrepõem-se a outros aspectos importantes e inerentes ao processo de ensino e aprendizagem. Outros estudos que busquem investigar aspectos envolvidos a dificuldades escolares e que proponham soluções para tais problemas são necessários para enriquecer a discussão sobre o tema. Pesquisas que relacionem o desenvolvimento do esquema corporal entre diferentes grupos de crianças, como escolares com e sem dificuldade de aprendizagem, ou que comparem outros aspectos do desenvolvimento motor também podem contribuir para melhor compreender a participação dos componentes motores nas habilidades cognitivas.

\section{Referências}

Amaro, K. N. (2010). Intervenção motora para escolares com dificuldade de aprendizagem. Dissertação de Mestrado, Universidade do Estado de Santa Catarina, Florianópolis, Santa Catarina.

Amaro, K. N., Brusamarello, S, Campos, F. C. G., \& Corazza, T. D. M (2008). Análise da organização temporal em escolares com dificuldade de aprendizagem. . Rev. Digital EFDeportes, 14(127), 1-1. Recuperado: 21 ago. 2008. Disponível: www.efdeportes.com/ efd127/organizacao-temporal-em-escolares-com-dificuldade-deaprendizagem.htm.

Amaro, K. N., Xavier, R. F. C, Corazza, T. D. M., Brusamarello, S, \& Rosa Neto, F. (2009). Desenvolvimento motor em escolares com dificuldade de aprendizagem. Rev. Digital EFDeportes, 14(133), 1-1. Recuperado: 21 mai. 2005. Disponível: http://www.efdeportes. $\mathrm{com} /$

Batistella, P. A. (2001.) Estudo de parâmetros motores em escolares com idade de 6 a 10 anos da cidade de Cruz Alta- RS. Dissertação de Mestrado, Universidade do Estado de Santa Catarina, Florianópolis, Santa Catarina.

Beresford, H, Queiroz, M., \& Nogueira, A. B. (2002). Avaliação das relações cognitivas e motoras na aquisição instrucional das habilidades para a aprendizagem da língua escrita. Revista Ensaio: avaliação política pública educacional, 10(37), 493-502.

Caetano, M. J. D., Silveira, C. R. A, \& Gobbi, L. T. B. (2005). Desenvolvimento Motor de Pré-escolares no Intervalo de 13 meses. Rev. Cineantropom. Desempenho Humano, 7(2), 5-13. 
Costa, S. H. (2001). Perfil motor de crianças der 05 a 14 anos com dificuldade na aprendizagem. Monografia de Graduação, Universidade do Estado de Santa Catarina, Florianópolis, Santa Catarina.

Capovilla, A. G. S., Capovilla, F. C., Trevisan, B., \& Rezende, M. C. A. (2006). Natureza das dificuldades de leitura em crianças brasileiras com dislexia do desenvolvimento. Revista Acolhendo a Língua Portuguesa: A alfabetização em foco, 1(1), p. 6-18.

Crippa, L. R., \& Souza, J. M. (2002). Estudo de parâmetros motores em pré-escolares: organização espacial, organização temporal e esquema corporal [Resumo]. Em R.J. Krebs (Org.), Encontro Latino-Americano para Estudos da Criança: Desenvolvimento Infantil, Suplemento (p.60). Florianópolis: UDESC- CEFID.

Cratty, B. J. (1964) Movement behavior and motor learning. Philadelphia: Lea and Febiger.

Ferreira, L. F., Nascimento, R. O., Apolinário, M. R., \& Freudenheim, A. M. (2006). Desordem da coordenação do desenvolvimento. Motriz, 12(3), 283-292.

Fiates, M. P. (2001). Estudo da relação entre o desenvolvimento psicomotor e as dificuldades na aprendizagem de um grupo de crianças de 4 a 7 anos. Monografia de Especialização, Universidade do Estado de Santa Catarina, Florianópolis, Santa Catarina.

Fonseca, V. (1983). Psicomotricidade. São Paulo: Martins Fontes.

Franceschi, C. R. (1997). Perfil motor em praticantes precoces de futebol de salão. Monografia de Graduação, Universidade do Estado de Santa Catarina, Florianópolis, Santa Catarina.

Freitas, N. K. (2008). Esquema corporal, imagem visual e representação do próprio corpo: questões teórico-conceituais. Ciências e Cognição, 13(3), 318-324.

Gallahue, D. L. (2000). Educação Física desenvolvimentista. Cinergis, 1(1), 7-17.

Gallahue, D. L., \& Ozmun, J. C. (2005). Compreendendo o desenvolvimento motor: bebês, crianças, adolescentes e adultos. São Paulo: Phorte.

Harrow, A. (1983). Taxionomia do desenvolvimento psicomotor: manual para elaboração de objetivos comportamentais em educação física. (M. A. V. Almeida, Trad.) Rio de Janeiro: Globo. (Trabalho original publicado em 1972)

Maturana, L. (2004). Imagem corporal: noções e definições. Rev. Digital EFDeportes, 10(71), 1-1. Recuperado: 21 mai. 2010. Disponível: http://www.efdeportes.com/

Medeiros, P. C., Loureiro, S. R., Linhares, M. B. M., \& Marturano, E. M. (2000). A Auto-Eficácia e os Aspectos Comportamentais de Crianças com Dificuldade de Aprendizagem. Psicologia: Reflexão e Crítica, 13(3), 327-336.

Medina, J., Rosa, G. K. B., \& Marques, I. (2006). Desenvolvimento da organização temporal de crianças com dificuldades de aprendizagem. Revista da Educação Física/ UEM, 17(1), 107116.

Medina-Papst, J., \& Marques, I. (2010). Avaliação do desenvolvimento motor de crianças com dificuldades de aprendizagem. Revista Brasileira de Cineantropometria e Desempenho Humano, 12(1), 36-42.

Melo, R. R. F. (1997) O perfil motor de alunos atletas de 8 a 10 anos que praticam regularmente natação. Monografia de Graduação, Universidade do Estado de Santa Catarina, Florianópolis, Santa Catarina.

Mello, L. M., Poeta, L. S., \& Rosa Neto, F. (2003). Aptidão motora em escolares com hiperatividade (TDAH) do ensino fundamental de Florianópolis/SC [Resumo]. Revista de Educação Física- UNESP, Suplemento,9(1), 164.

Neto, F. R., Almeida, G. M. F., Caon, G., Ribeiro, J., Caram, J. A., \& Piucco, E. C. (2007). Desenvolvimento motor de crianças com indicadores de dificuldades na aprendizagem escolar. Revista Brasileira de Ciência e Movimento, 15(1), 45-51.

Pacheco, L. M. B. (2005). Diagnóstico de Dificuldade de Aprendizagem?! Temas em Psicologia da SBP, 13(1), 45-51.

Pazin, J. (2001). Avaliação do desenvolvimento motor, físico e psicossocial em escolares. Dissertação de Mestrado, Universidade do Estado de Santa Catarina, Florianópolis, Santa Catarina.

Poeta, L. S. (2005). Avaliação e intervenção motora em escolares com indicadores de Transtorno do Déficit de Atenção/Hiperatividade (TDAH). Dissertação de Mestrado, Universidade do Estado de Santa Catarina, Florianópolis, Santa Catarina.

Rosa Neto, F. (2002). Manual de avaliação motora. Porto Alegre: Artmed.

Rosa Neto, F., Poeta, L. S., Coquerel, P. R. S., \& Silva, J. C. (2004). Avaliação motora em escolares com problemas na aprendizagem escolar - programa de Psicomotricidade. Temas sobre Desenvolvimento, 13(74), 19-24.

Rosa Neto, F., Poeta, L. S, Silva, J. C., \& Silva, M. B. B. (2006). Intervenção psicomotora: projeto de extensão universitária. Rev. Iberoamericana de Psicomotricidad y Técnicas Corporales, 7(26), 197-204

Scagliotta, E. G. (1970). Inicial learning assessment. San Rafael, Calif: Academic Therapy Publications.

Silva, C. A., Rosa Neto, F., Almeida, G. M. F., Amaro, K. M., \& Silva, M. B. B. (2006). A importância da avaliação motora em escolares. 
Rev. Iberoamericana de Psicomotricidad y Técnicas Corporales, 7(26), 137-146.

Tavares, M. C. G. C. (2003). Imagem corporal: conceito e desenvolvimento. São Paulo: Manole.

Veyer, P, \& Destrooper, J. (1979) La dinámica de la acción educativa en los niños inadaptados. Barcelona: Científico- Médica.
Vieira, L. F., Santos, V. C., Vieira, J. L. L., \& Oliveira, A. A. B. (2004). Análise da aprendizagem perceptivo-motora de crianças de 7 e 8 anos da rede de ensino público de Maringá - PR. Revista da Educação Física/UEM, 15(2), 39-48.

\section{Sobre os autores}

Francisco Rosa Neto (franciscorosaneto@terra.com.br)

Universidade do Estado de Santa Catarina

Kassandra Nunes Amaro (kakitanunes@gmail.com)

Universidade do Estado de Santa Catarina em Florianópolis

Daniela Bosquerolli Prestes (danibprestes@hotmail.com)

Universidade do Estado de Santa Catarina em Florianópolis

Claudia Arab (cau_arab@hotmail.com)

Universidade do Estado de Santa Catarina em Florianópolis

\section{Endereço para correspondência:}

Universidade do Estado de Santa Catarina - UDESC

Centro de Ciências da Saúde e do Esporte - CEFID

Laboratório de Desenvolvimento Humano - LADEHU

Núcleo de Avaliação e Intervenção Motora - NAIM

Rua Pascoal Simone, 358 - Coqueiros, Florianópolis.

CEP: $88080-350$ 